Nachruf

Z. Epileptol. 2021 · 34:239-240 https://doi.org/10.1007/s10309-021-00406-3 Angenommen: 9. Februar 2021

Online publiziert: 2. März 2021

(c) Springer Medizin Verlag GmbH, ein Teil von Springer Nature 2021
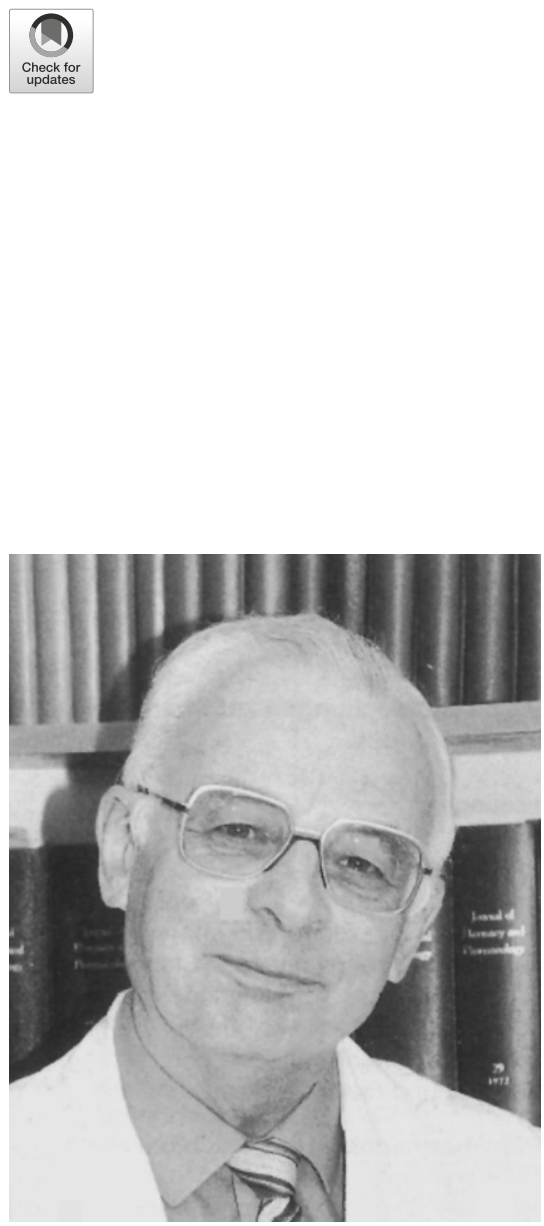

Abb. 1 \ Hans-Hasso Frey ca. 1992. (Foto:W. Löscher)

Am 28.12.2020 ist Prof. Dr. med. vet. Hans-Hasso Frey (• Abb. 1) im Alter von 93 Jahren in Neustadt in Holstein verstorben. Er war eine außergewöhnliche Persönlichkeit als international renommierter Wissenschaftler, akademischer Lehrer und engagierter Hochschulpolitiker.

In Leipzig geboren, wuchs Frey in Hannover auf und studierte dort von 1946 bis 1950 Veterinärmedizin. Sein Interesse an der Pharmakologie zeigte sich damals schon bei der Auswahl seiner Doktorarbeit über die lumbosakrale Epiduralanästhesie beim Hund, mit der er 1951 promoviert wurde. Nach einem kurzen Intermezzo in der tierärztlichen Praxis fiel 1953 seine endgültige Entscheidung für

Wolfgang Löscher ${ }^{1} \cdot$ Heidrun Fink ${ }^{2} \cdot$ Hartmut Wei ${ }^{3} \cdot$ Günter Krämer ${ }^{4}$

'Forschungsgruppe Epilepsie, Institut für Pharmakologie, Toxikologie und Pharmazie, Tierärztliche Hochschule Hannover, Hannover, Deutschland

${ }^{2}$ Institut für Pharmakologie und Toxikologie, Fachbereich Veterinärmedizin, Freie Universität Berlin, Berlin, Deutschland

${ }^{3}$ Fachgebiet Biometrie, Fachbereich Veterinärmedizin, Freie Universität Berlin, Berlin, Deutschland

${ }^{4}$ Neurozentrum Zürich, Zürich, Schweiz

\title{
Nachruf auf Professor Dr. Hans-Hasso Frey (21.09.1927-28.12.2020)
}

die Pharmakologie mit Annahme einer Stelle als wissenschaftlicher Mitarbeiter am Pharmakologischen Institut der Universität Hamburg. Nach einem kurzen Zwischenaufenthalt 1956 am Pharmakologischen Institut der Tierärztlichen Fakultät der Universität München wechselte er noch im selben Jahr zu Richard Völker (1896-1981) an das Pharmakologische Institut der Tierärztlichen Hochschule Hannover (TiHo) zurück. Zwei Jahre später habilitierte er sich mit einer grundlegenden Arbeit über den Stoffwechsel von Kurznarkotika aus der Reihe der Barbitursäurederivate und blieb als Privatdozent noch einige Jahre an diesem Institut. In diese Zeit reichen seine ersten Untersuchungen über Antiepileptika zurück (z. B. [1]).

Im Jahr 1961 übernahm Frey die Leitung der pharmakologischen Abteilung der dänischen Pharmafirma Leo Pharmaceutical Products in Ballerup. Dort war er unter anderem mit immunologischen Fragestellungen und der Entwicklung von Diuretika befasst. Das hochwirksame Schleifendiuretikum Bumetanid wurde maßgeblich unter seiner Leitung entwickelt. Interessanterweise wird dieser Wirkstoff aktuell auch bei neonatalen Anfällen eingesetzt [2,3]. Das Hauptinteresse von Frey galt ohnehin weiterhin der ZNS-Pharmakologie. Während seiner Zeit in Dänemark hielt er weiter Vorlesungen an der TiHo und wurde 1964 zum außerplanmäßigen Professor ernannt.
Von den 1968 gleichzeitig an ihn ergangenen Rufen auf den Lehrstuhl für Pharmakologie, Toxikologie und Pharmazie der Tierärztlichen Fakultät der Universität München und den Lehrstuhl für Pharmakologie und Toxikologie des Fachbereichs Veterinärmedizin der Freien Universität (FU) Berlin nahm er 1969 den Ruf nach Berlin an. Einen erneuten Ruf auf den Lehrstuhl in München lehnte er $1972 \mathrm{ab}$ und leitete bis zu seiner Emeritierung 1996 über 25 Jahre das Institut für Pharmakologie und Toxikologie am Fachbereich Veterinärmedizin der FU Berlin.

Frey setzte in Berlin seine Studien zur ZNS-Pharmakologie und über Diuretika fort und entwickelte das Berliner Institut mit Ideenreichtum, forscherischem Weitblick und unter Bewältigung eines riesigen Arbeitspensums trotz der beengten räumlichen Verhältnisse $\mathrm{zu}$ einer der forschungsaktivsten Einrichtungen des Fachbereichs Veterinärmedizin. Die Epilepsieforschung rückte bald in den Mittelpunkt, wobei sich seine Arbeitsgruppe, eingebunden in intensive interdisziplinäre Zusammenarbeit, mit grundlegenden Arbeiten zur Pharmakologie von Antiepileptika hohes internationales Ansehen erwarb, das sich unter anderem daran ermessen lässt, dass ihr eine tragende Rolle in einem langjährig von der DFG geförderten Schwerpunktprogramm zukam. Im Jahr 1985 gab Frey zusammen mit Dieter Janz (1920-2016) ein Standardwerk über An- 
tiepileptika heraus [4], ein Jahr später war er federführender Herausgeber eines Buches zur Toleranzentwicklung unter und Nebenwirkungen von Antiepileptika [5]. Zusammen mit Janz initiierte Frey an der FU in Berlin ein regelmäßiges epileptologisches Kolloquium, an dem alle an Epilepsie interessierten Berliner Wissenschaftler teilnahmen, um neue Ergebnisse aus der Forschung zu präsentieren und auszutauschen.

Das Ergebnis seiner breiten und intensiven wissenschaftlichen Tätigkeit sind über 200 wissenschaftliche Originalarbeiten und Handbuchartikel sowie zahlreiche Vorträge auf nationalen und internationalen Tagungen. Mit stets neuen Denkansätzen und dem Mut zu neuen Methoden prägte er viele junge Kollegen. Von seinem Wissen und Können profitierten nicht nur seine Schüler und Mitarbeiter, sondern auch Fachkollegen, die das Glück hatten, mit ihm enger zusammenzuarbeiten. Aufgrund seiner hohen fachlichen Kompetenz war er auch ein gefragter Experte in zahlreichen Kommissionen, beispielsweise als Mitglied der Arzneibuchkommission und des Ausschusses für Standardzulassungen beim früheren Bundesgesundheitsamt (BGA). Er war langjähriger Vorsitzender des wissenschaftlichen Beirats des Instituts für Arzneimittel des BGA sowie Mitglied der Zulassungs- und Aufbereitungskommission für Tierarzneimittel am BGA.

Neben der Forschung war die Lehre immer ein besonderes Anliegen von Hans-Hasso Frey. Er hat vielen Studentengenerationen den Einstieg und das Verständnis für Pharmakologie und die Voraussetzungen für einen gezielten und gewissenhaften Umgang mit Arzneimitteln vermitteln können, wobei er wie kaum ein anderer über weite Bereiche aus dem Fundus eigener experimenteller Erfahrungen schöpfen konnte. Anfang der 1990er-Jahre begann er mit einem von uns (Wolfgang Löscher), das erste deutschsprachige Lehrbuch der Pharmakologie und Toxikologie für die Veterinärmedizin zusammenzustellen, das 1996 erstmals erschien [6] und inzwischen in der 4. Auflage als Standardwerk dieses Faches gilt. Darüber hinaus fand Frey immer noch Zeit für hochschulpoli- tisches Engagement und die Übernahme verschiedener Aufgaben im Fachbereich und in der universitären Selbstverwaltung (1976-1979 Vorsitzender bzw. Sprecher des Fachbereichs und 1981-1983 Dekan).

Hans-Hasso Frey war ein gesuchter Ratgeber, nicht nur wegen seiner großen Sachkunde, sondern auch wegen seiner, sicher nicht immer bequemen, unbestechlichen Kritik und Offenheit. Von den zahlreichen Auszeichnungen und Preisen sei hier stellvertretend der 1980 vom Epilepsie-Kuratorium in Bonn verliehene Alfred-Hauptmann-Preis erwähnt.

Nach seiner Emeritierung zog Frey mit seiner Frau Anni nach Neustadt in Holstein und damit in den geliebten Norden um. Nach dem frühen Tod seines Sohnes Matthias und, später, auch seiner Ehefrau zog er sich zunehmend zurück, blieb aber früheren Schülern und Kollegen ein interessierter Ansprechpartner und Freund. Er pflegte auch nach wie vor Kontakte zu Klinikern im In- und Ausland. Wir werden ihn vermissen und das Andenken an ihn in Ehren bewahren.

Wolfgang Löscher, Hannover

Heidrun Fink, Potsdam

Hartmut Weiß, Berlin

Günter Krämer, Zürich

\section{Korrespondenzadresse}

Prof. Dr. med. vet. Wolfgang Löscher

Forschungsgruppe Epilepsie, Institut für Pharmakologie, Toxikologie und Pharmazie, Tierärztliche Hochschule Hannover Bünteweg 17, 30559 Hannover, Deutschland wolfgang.loescher@tiho-hannover.de

\section{Literatur}

1. Frey H-H, Hahn I (1960) Untersuchungen über die Bedeutung des durch Biotransformation gebildeten Phenobarbital für die antikonvulsive Wirkung von Primidon. Arch Int Pharmacodyn 128:281-290

2. Pressler RM, Boylan GB, Marlow N et al (2015) Bumetanide for the treatment of seizures in newborn babies with hypoxic ischemic encephalopathy (NEMO): an open-label, dose finding, and feasibility phase 1/2 trial. Lancet Neurol 14:469-477

3. Soul JS, Bergin AM, Stopp C, Boston Bumetanide Trial Group (2021) A pilot randomized, controlled, double-blind trial of bumetanide to treat neonatal seizures. Ann Neurol 89:327-340

4. Frey H-H, Janz D (Hrsg) (1985) Antiepileptic drugs. Handbook of experimental pharmacology, Bd. 74. Springer, Berlin

5. Frey HH, Fröscher W, Koella WP, Meinardi H (Hrsg) (1986) Tolerance to beneficial and adverse effects of antiepileptic drugs. Workshop on tolerance to beneficial and adverse effects of antiepileptic drugs, Hamburg, Federal Republic of Germany, September 1985. Raven Press, New York

6. Frey H-H, Löscher W (Hrsg) (1996) Lehrbuch der Pharmakologie und Toxikologie für die Veterinärmedizin. F. Enke, Stuttgart (letzte Auflage als Mitherausgeber: Frey H-H, Löscher W, Hrsg. Lehrbuch der Pharmakologie und Toxikologie für die Veterinärmedizin. 3., vollständig überarbeitete Auflage. Stuttgart, F. Enke 2010) 